\title{
Breast Cancer Detection using Local Binary Patterns
}

\author{
S. Naresh \\ M.Tech \\ Department of CSE \\ GMR Institute of Technology \\ Rajam, India
}

\author{
S. Vani Kumari \\ Asst. Professor \\ Department of CSE \\ GMR Institute of Technology \\ Rajam, India
}

\begin{abstract}
Breast cancer is a leading cause of cancer type for death among women in most of popular countries, breast cancer detection is important and challenging role in worldwide to save women's life. Due to inexperience to detect cancer, the doctors and radio logistic can miss the abnormality, which leads to death. Mammography is the most used method for breast cancer detection used by the radiologists. In this experiment, the MIAS (Mammogram Image Analysis Society) database is used and the MIAS database consists of normal and abnormal type of 322 mammograms. The pre-processing is most important step to capture quality mammogram image for next study and processing in mammogram analysis. Texture analysis plays important role to identify normal and abnormal types. Texture feature extraction can be done by local binary patterns (LBP) operator and by using LBP we can consider only sign parameters, it may loss the some texture information. The local binary pattern is a rotation invariant approach for the texture analysis. In this experiment famous completed LBP (CLBP) method used for extracting texture features. Completed LBP considering the sign, magnitude and centre gray level values. By using the joint or hybrid distributions combine CLBP_Sign, CLBP_Magnitude and CLBP_Center gray level values.LBP is one type of Completed LBP for texture analysis, advantage of CLBP is rotation invariant. Finally extracted texture features can be trained and classified by using the SVM classifier for identifying the normal and abnormal cancer type.
\end{abstract}

\section{Keywords}

Breast Cancer; Mammogram; Pre-processing; Rotation invariant; CLBP; SVM Classification

\section{INTRODUCTION}

Breast cancer is the second greatest cause of death in women, especially for women age greater than 35 year. In UK year of 2011, the cancer research UK estimated the occurrence of 50,285 new cases of breast cancer will be diagnosed among women. In 2012, 11,716 UK women died due to breast cancer. In 2014 an American cancer society estimate 2, 32,670 among 40,000 women's died due to breast cancer. Due to the late detection of breast cancer women died rate will be gradually increased. Mammography [1] is the best method for early detection of breast cancer abnormalities such as masses, microcalcifications, asymmetries, architectural distortions. Mammography screening has an effective in reducing breast cancer mortality rates. Identifying masses, asymmetries are difficult for radiologist due to the inexperience, they may give wrong output because the mammograms are very sensitive to identify. CAD (Computer -aided diagnosis) technology can improve the performance of radiologistics. The CAD technique can reduce the occurrence of errors committed by the specialist. CAD system can classify the normal, abnormal cases and benign, malignant cases from mammograms by extracting the texture features and classify the features.

Input to CAD system MIAS (Mammographic Image Analysis Society) database is taken and MIAS database consists of 322 images (normal and abnormal) from 161 patients. The mammographic images have the artefacts and digital noise. Pre-processing is requiring for better output results, in this process eliminate the unnecessary noisy data and enhance the contract of poor quality images.. Texture feature extraction is done by using the complete local binary pattern method and the extracted can classify using SVM classifier, identify the normal and normal cases.

\section{PREPROCESSING}

The objective of preprocessing [3] is remove an irrelevant noisy and improve the quality of an image, eliminate an unwanted parts in mammogram background and make it ready for further processing. Preprocessing is very important step in mammogram image analysis and enhances the image quality and influence the result, improve the accuracy of result and play the major role to identify abnormality.

Morphological operations [6] are used to remove the noisy data, enhance and contrast the input image. Morphological operators are those applied on the binary image, before convert the gray image into binary image. Four morphological operations are used to improve the image for further process; they are Erosion, Dilation, Top-Hat and Bottom-Hat. Dilation operator can gradually enlarge the boundaries of regions of foreground pixels (i.e. white pixels) of binary image. Erosion operator can erode away the boundaries of regions of foreground pixels (i.e. white pixels,), thus areas of foreground pixels shrink in size, and holes within those areas become larger. Detailed information of image can achieve by using Top-Hat operator, the brightest part of an image become highlight. Bottom-Hat operator can used of highlight the black spot in a white background. Erosion and Dilation operators can used for reduce the noisy, Top-Hat and Bottom-Hat operators can used for enhance and contrast the image. Contrast image can achieves by subtracting the Bottom-Hat image from Top-Hat and initial image. Eliminate the muscle part by using the k-means.

\section{BRIEF REVIEW OF LBP}

Local Binary Patterns (LBP) is a method for texture feature extraction mostly used for recognition techniques. The extracted features are useful for classifying breast cancer abnormality in mammograms. Given a pixel in the image, an LBP code is computed by comparing it with its neighbours. There are two user defined parameter for input to LBP, they are $\mathrm{P}$ (number of neighbours) and $\mathrm{R}$ (radius of comparisons). 


$$
L B P_{P, R}=\sum_{P=0}^{P-1} s\left(g_{p}-g_{c}\right) 2^{p}, s(x)=\left\{\begin{array}{l}
1, x \geq 0 \\
0, x<0
\end{array}\right.
$$

Where $g p$ is the value of its neighbours, $g c$ is the gray value of the central pixel, $\mathrm{P}$ is the total number of involved neighbours and $R$ is the radius of the neighbourhood. First fix the center value, compare the center value with neighbour values if neighbour value higher than the center value then assign 1 to that position otherwise assign 0 value. The main advantage by using LBP is rotation invariant feature extraction and finally grouping the extracted features in histogram. By using (1) we can calculate the sign parameter for neighbourhood pixel.

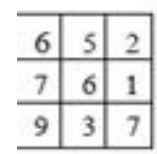

(a)

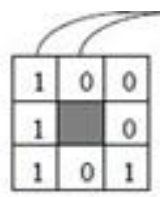

(b)

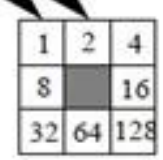

(c)

\begin{tabular}{|c|c|c|}
\hline 1 & 0 & 0 \\
\hline 8 & & 0 \\
\hline 32 & 0 & 128 \\
\hline
\end{tabular}

(d)

\section{Fig.1. Local Binary Pattern Operation}

The above illustrated example can take the $3 \times 3$ neighbourhood Fig. 1(a) is threshold at the value of the center pixel, the values of the pixels in the threshold neighbourhood Fig. 1(b), corresponding binomial weights Fig. (1c), multiplied binominal corresponding pixels Fig. 1(d), finally the output value $169(1+8+32+128)$ comes after combining the eight neighbour values.

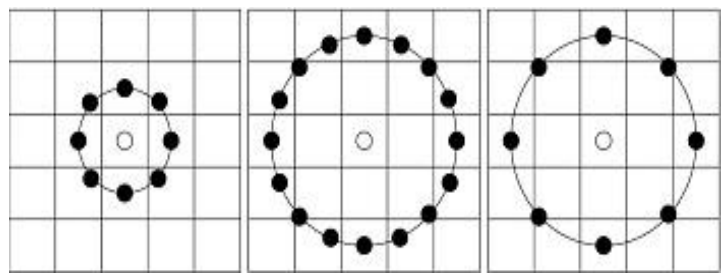

Fig. 2. The circular $(8,1),(16,2)$ and $(8,2)$ neighbourhoods

Fig. 2 illustrate the different $P, R$ values given as input to the LBP, the pixel values are bilinear interpolated whenever the sampling point is not in the center of a pixel. Ojala et al. proposed the Local Binary Pattern (LBP) for rotation invariant texture classification. LBP can also useful to detect the face reorganization, shape localization and dynamic texture recognition.

In many texture analysis applications if input image rotate, the features that are robust or invariant. The LBP patterns are circularly sampling around the center pixel, rotation of the input image has two effects: the sampling points on the circle surrounding the center point are rotated into a different orientation each local neighbourhood is rotated into other pixel location, and within each neighbourhood. It is also possible rotation invariance by grouping the extracted features in histograms.

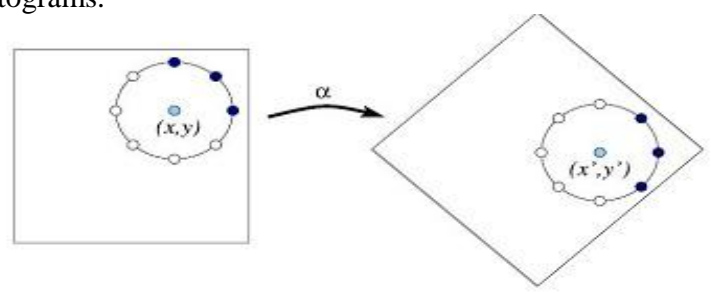

Fig. 3. Effect of image rotation on points in circular neighbourhoods
In Fig. 3, let $U_{P}(n, r)$ denote a specific uniform LBP pattern. The pair $(n, r)$ specifies a uniform pattern, $r$ is the rotation of the pattern (column number in Fig. 3) and $n$ is the number of 1bits in the pattern. If the neighbourhood has $\mathrm{P}$ sampling points, $\mathrm{n}$ varying the value from 0 to $\mathrm{P}+1, \mathrm{n}=\mathrm{P}+1$ maximum of nonuniform patterns. Let $I^{\alpha^{o}}$ denote the rotation of image $I(x, y)$ by $\alpha$ degrees, If the rotations are limited to integer multiples of the angle between two sampling points, i.e. $\alpha=a \frac{360^{\circ}}{P}$; $a=0,1, \ldots . P-1$, this rotates the sampling neighbourhood by exactly $a$ discrete steps. Therefore the uniform pattern $U_{P}(n, r)$ at point $(x, y)$ is replaced by uniform pattern $U_{P}(n, r+a \bmod P)$ at point $(x, y)$ of the rotated image.

\section{COMPLETED LBP}

In Completed Local Binary Pattern [3], a local region can be represented as its centre gray level and local difference sign magnitude transform LDSMT). The LDSMT decomposed into sig $\mathrm{n}$ and magnitude components. The CLBP includes the CLBP_C, CLBP_S, and CLBP_M Operators. CLBP_S and CLBP_M are used to code the sign and magnitude components respectively; CLBP_C is used to code the centre gray level after global thresholding.

\begin{tabular}{|c|c|c|}
\hline 45 & 15 & 68 \\
\hline 65 & 50 & 70 \\
\hline 90 & 30 & 10 \\
\hline
\end{tabular}

(p)

\begin{tabular}{|l|l|l|}
\hline-1 & -1 & 1 \\
\hline 1 & & 1 \\
\hline 1 & -1 & -1 \\
\hline
\end{tabular}

(r)

\begin{tabular}{|c|c|c|}
\hline-5 & -35 & 18 \\
\hline 15 & & 20 \\
\hline 40 & -20 & -40 \\
\hline
\end{tabular}

(q)

\begin{tabular}{|l|l|l|}
\hline 5 & 35 & 18 \\
\hline 15 & & 20 \\
\hline 40 & 20 & 40 \\
\hline
\end{tabular}

(s)
Fig. 4(p) A 3*3 sample example; Fig. 4(q) the local differences; Fig. 4(r) the sign and Fig. 4(s) magnitude components

Fig 4 shows an example, Fig. 4(p) is the original $3 * 3$ local structure with central pixel 50 . The difference vector Fig. 4(q) is $[3,9,-13,-16,-15,74,39,31]$. After LDSMT, the sign vector Fig. $4(\mathrm{r})$ is $[-1,-1,1,-1,-1,1,1,1]$ and the magnitude vector Fig. $4(\mathrm{~s})$ is $[5,35,18,20,40,20,40,15]$. The equalling LBP 8-bit string is " 00110011 " ("- 1 " is coded as " 0 "), the LBP uses only the sign vector and the CLBP is use the sign and magnitude vectors.

The CLBP_S operator can be given by

$C L B P_{S_{P, R}}=\sum_{P-0}^{P-1} S\left(g_{p}-g_{c}\right) 2^{P}$

Where $S(x)=\left\{\begin{array}{l}1, x \geq 0 \\ 0, x<0\end{array}, \mathrm{~g}_{\mathrm{c}}\right.$ is the gray value of the center pixel, $g_{p}$ is the value of its neighbours, $P$ is the number of involved neighbours.

The CLBP_M operator can be defined by 
$C L B P_{M_{P, R}}=\sum_{p=0}^{P-1} t\left(m_{P}, c\right) 2^{P}$

Where $t(x, c)=\left\{\begin{array}{l}1, x \geq 0 \\ 0, x<0\end{array}\right.$, and $\mathrm{c}$ is the threshold value, here it is set as $m_{p}$ which is the mean value for the whole image. The CLBP_C can be defined by

$$
C L B P_{C_{P, R}}=t\left(g_{c}, C_{1}\right)
$$

Where $C_{1}$ is the threshold here it is set as average gray level of the image.

The Fig. 5 is the framework for CLBP, the initially divide the image into centre gray level and LDSMT (Local Difference Sign Magnitude thresholding). From the LDSMT again calculate sign, magnitude vectors. Finally combine the CLBP_S, CLBP_M and CLBP_C features by using join or concatenation operation and form CLBP histogram.

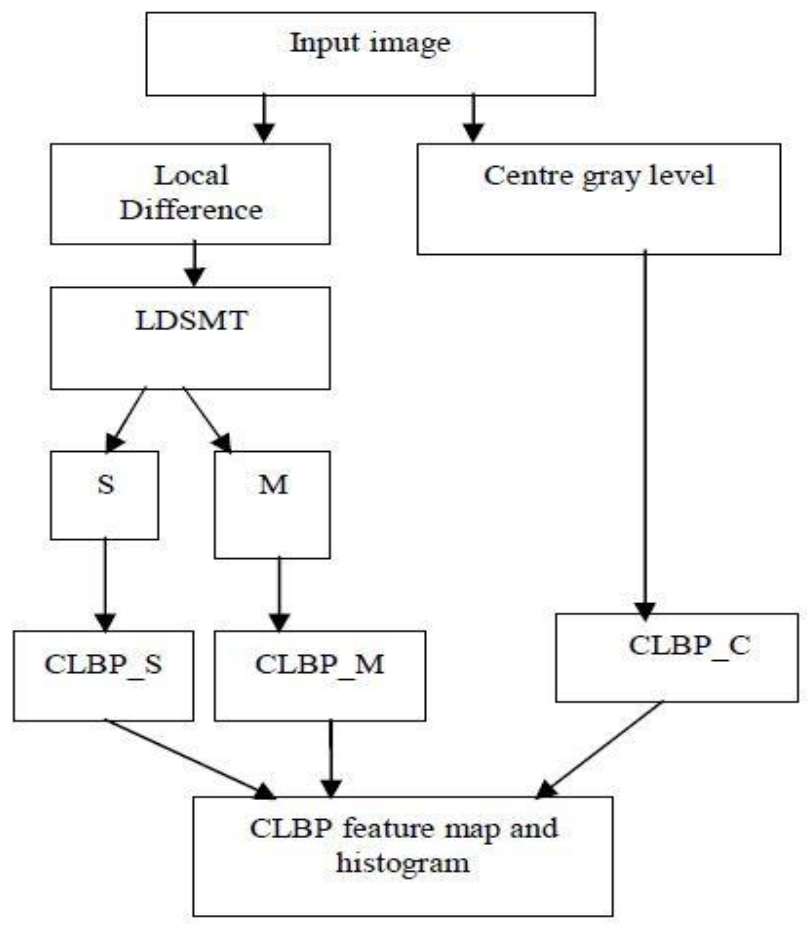

Fig 5: framework for CLBP

\section{RESULTS AND DISCUSSIONS}

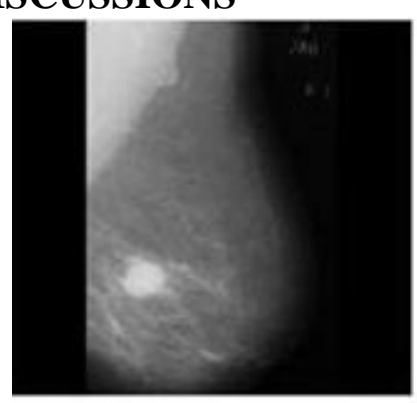

(a)

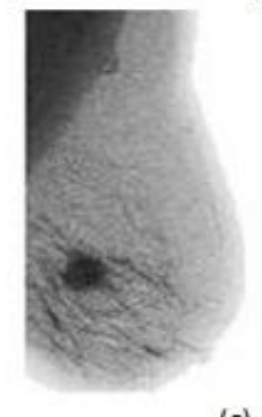

(c)

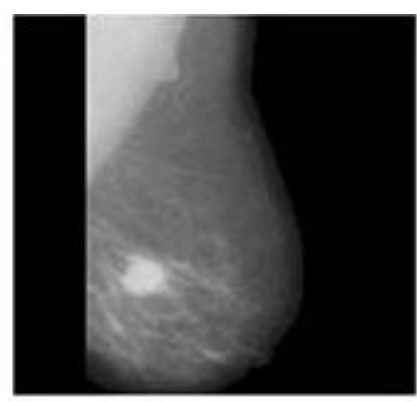

(b)

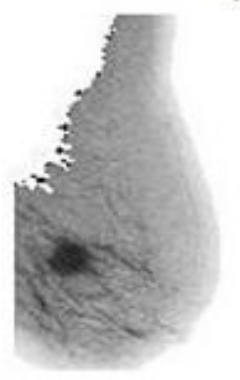

(d)

Fig. 6 (a) Initial image; Fig. 6(b) Eliminate noisy and unnecessary data; Fig. 6(c) Image after enhancement using Top-Hat and Bottom-Hat; Fig. 6(d) Muscle removing image 
The texture feature extracting using the Completed Local Binary Pattern is performed in the mammogram image taken from MIAS database. Image preprocessing and enhancement operation can be done by using morphological operators.

In Fig. 6(a) the mammogram image taken input from the MIAS database, Fig. 6(b) is an image after applying the dilation, erosion morphological operations, Fig. 6(c) is an image after applying Top-Hat and Bottom-Hat morphological operations for image enhancement and contrast and Fig. 6 (d) is an image removing muscle part that influence the result, by using k-means [5] algorithm muscle part can remove.

The Fig. 7 comes after applying the CLBP operation for extracting the features, by achieving histogram image by grouping the extracted CLBP features. Here the image is in gray level. So the histogram value will change from 0 to 255 . So there are 256 bins in the histogram.

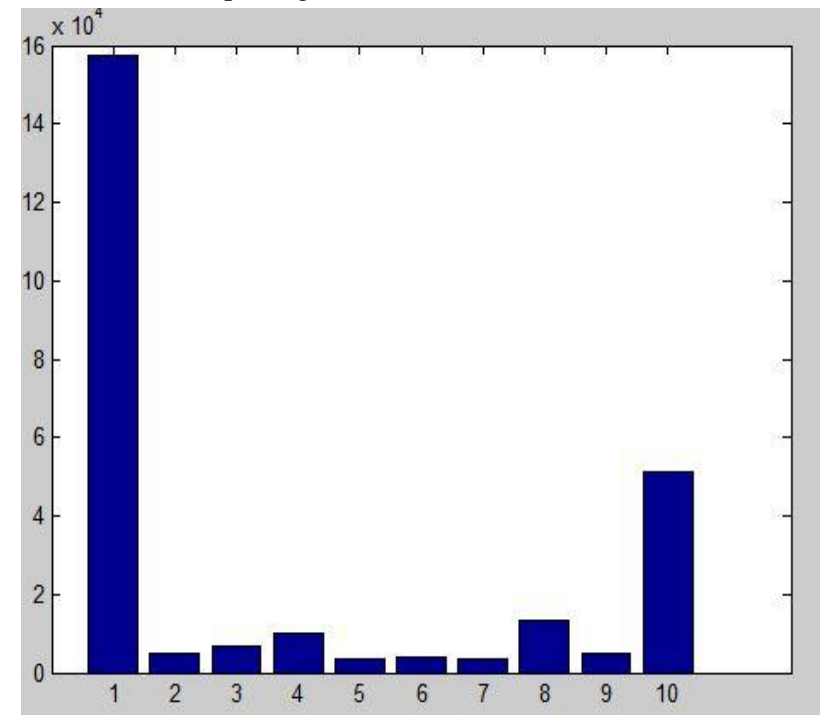

Fig 7: CLBP histogram

\section{CONCLUSION}

By using Local Binary Pattern (LBP) we can extracted the texture features, in LBP we can consider only sign parameter. By using only sign parameter loose the more image local information. But CLBP consider the both sign and magnitude parameters. By using CLBP we can get the robust results for breast cancer detection and CLBP is used for most recognition techniques.

\section{REFERENCES}

[1] Eanes Torres Pereira and Sidney Pimentel Eleutério, "Local Binary Patterns Applied to Breast Cancer Classification in Mammographies," on RITA _ Volume 21 _ Número 2 _ 2014.

[2] Zhenhua Guo and Lei Zhang, "A Completed Modelling of Local Binary Pattern Operator for Texture Classification," on IEEE Transactions on Image Processing.

[3] D.Narain Ponraj and M.Evangelin Jenifer, "A Survey on the Preprocessing Techniques of Mammogram for the Detection of Breast Cancer," on Journal of Emerging Trends in Computing and Information Sciences VOL. 2, NO. 12, December 2011 ISSN 2079-8407.
[4] Anupa Maria Sabu, D.Narain Ponraj\& Poongodi, "COMPLETED LBP BASED TEXTURE ANALYSIS IN MAMMOGRAM,", International Journal of Electronics Signals and Systems (IJESS), ISSN: 22315969, Vol-3, Iss-1, 2013

[5] Jawad Nagi, Sameem Abdul Kareem, "Automated Breast Profile Segmentation for ROI Detection Using Digital Mammograms," 2010 IEEE EMBS Conference on Biomedical Engineering \& Sciences (IECBES 2010), Kuala Lumpur, Malaysia, 30th November - 2nd December 2010.

[6] Abdelali Elmoufidi, Khalid El Fahssi, Said JaiAndaloussi, Abderrahim Sekkaki,," Detection of Regions of Interest in Mammograms by Using Local Binary Pattern and Dynamic K-Means Algorithm," Image and video processing theory and applications Vol. 1, No. 1, 30 April 2014 ISSN: 2336-099

[7] J. Anitha and J. Peter. "A wavelet based morphological mass detection and classification in mammograms". In Machine Vision and Image Processing (MVIP), 2012 International Conference on, pages 25-28, 2012. 